\title{
Different surgical approaches for nasal-orbit- ethmoidal fractures: two cases report
}

\begin{abstract}
The aim of this report is to provide a vision of the approaches used to resolve NasalOrbit-Ethmoidal (NOE) fractures, a purpose of two cases treated by two different types of approaches. The first case corresponds to a patient with a complex Nasal-Orbit-Ethmoidal fracture, treated by the use of pre- existing lacerations and with a transconjunctival approach with canthotomy and lateral extension, to access an orbital zygomatic fracture. The second case corresponds to a patient with Nasal-Orbit-Ethmoidal Fracture treated by the use of a conventional Coronal approach. Both cases were treated by the maxillofacial surgery team at Hospital El Carmen, Santiago Chile, and prior evaluation by Neurology, ophthalmology and anesthesiology was necessary due to the magnitude and extent of the trauma, ruling out neurological and ophthalmological compromise. Finally, it can be concluded from these cases that both, coronal approach and the use of prior lacerations are a viable alternative for access to the fractured area, obtaining good visibility, access and aesthetic results. We also conclude that fractures of the Nasal-Orbit-Ethmoidal complex are a great challenge for the maxillofacial surgeon due to its high morbidity, added to the aesthetic and psychological component associated with them and functional alterations. This is why the correct and timely management of these fractures is key to minimize complications and sequelae that they have
\end{abstract}

Keywords: maxillofacial trauma, nasal, orbit-ethmoidal fractures, coronal approach, laceration
Volume 10 Issue 5 - 2019

\section{Salvador Valladares Pérez, Diego Bustamante Correa,Gerson Sepúlveda Troncoso \\ School of Dentistry, El Carmen Metropolitan Clinical Hospital, Pontifical Catholic University of Chile, Chile}

Correspondence: Diego Bustamante Correa, School of Dentistry, El Carmen Metropolitan Clinical Hospital, Pontifical Catholic University of Chile, Chile, Email d.bustac@gmail.com

Received: September 01, 2019 | Published: September 10, 2019

\section{Introduction}

Trauma is defined as damage with or without intention caused to the organism by a sudden exposure to energy sources that exceed its tolerance margin. In turn, facial skull trauma is described as one that affects the dentoalveolar complex, bony structures and soft tissues of the maxillofacial territory. ${ }^{1}$ The Nasal-Orbit-Ethmoidal Complex fractures cover the middle area of the midfacial third, being extended fractures when they have a bilateral commitment, with a high aesthetic compromise and difficult to handle, thus becoming a great challenge for maxillofacial surgeons. ${ }^{1}$ The Nasal-Orbit-Ethmoidal complex corresponds to a complex structure formed by the nasal bones, nasal septum, nasal-frontal process, ethmoids, papiraceous laminae of the lacrimal bone and sphenoid, so any alteration of these structures will have important functional and aesthetic consequences. ${ }^{3}$ Nowadays, there are several ways to approach these fractures, the coronal approach being the most used since it allows a correct access and visibility of the nasal frontal area. Despite this, this approach requires precision and surgical expertise. On the other hand, due to the area of the incision, hair less patients have a considerable aesthetic impact. ${ }^{4}$ Below are two cases of Nasal-Orbit-Ethmoidal fractures evaluated and treated by the maxillofacial surgery team at the El Carmen Metropolitan Hospital, Santiago, Chile. Both required neurological and ophthalmological evaluation, discarding commitment at that level. The first case corresponds to a patient with an extended fracture of the Nasal-Orbit-Ethmoidal complex in which an approach was made through pre-existing lacerations in the frontal nasal area, complementing it with a transconjunctival approach with extension and lateral canthotomy to access the lateral wall and floor of the right orbit. The second patient corresponds to a patient with a fracture of the frontal component and Nasal-Orbit-Ethmoidal which was approached by means of a coronal incision.

\section{Cases}

\section{Case I}

Male patient, 28 years old with complex trauma of the midfacial third. The clinical exam presents a complex wound that reaches the osseous plane, and a Nasal-Orbit-Ethmoidal fracture and right zygomatic orbital (Figure $1 \& 2$ ). The cause of the trauma was a direct hit in the fronto-Nasal region with a blunt object. Under general anesthesia and orotracheal intubation, the approach to the fracture zone is performed by means of the pre-existing lacerations in the nasofrontal area and a transconjunctival approach with lateral extension to access the floor and lateral wall of the right orbit (Figure $3 \& 4$ ). A correct reduction of the fractured fragments was achieved, returning the anatomy and correct functionality (Figure 5). Finally, the patient required 48 hours of hospitalization to observe his evolution and postoperative analgesic management and then continue with outpatient follow up (Figure 6).

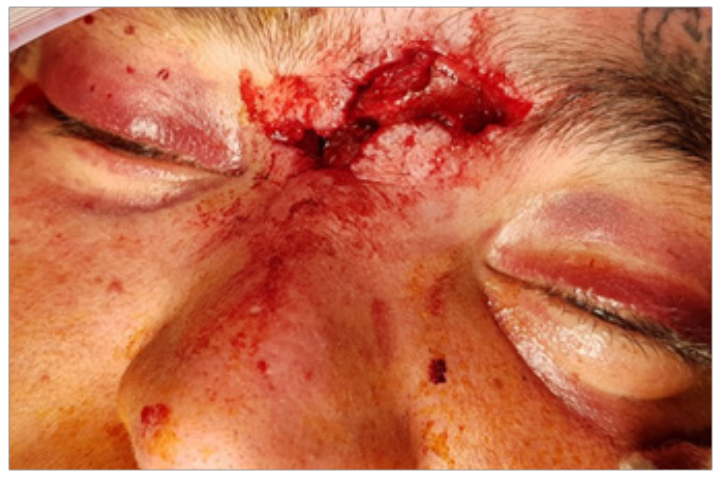

Figure I NOE fracture. 


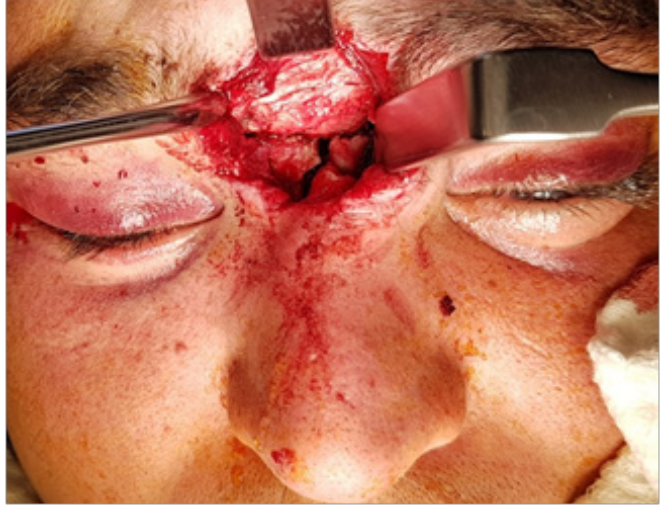

Figure 2 Right zygomatic orbital.

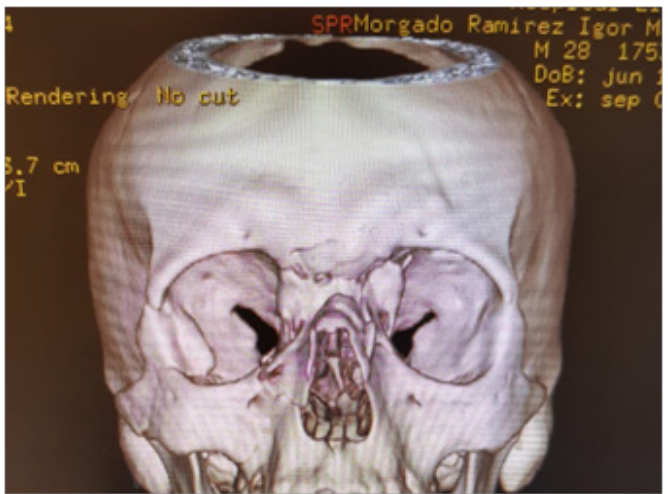

Figure 3 Lacerations in the nasofrontal area.

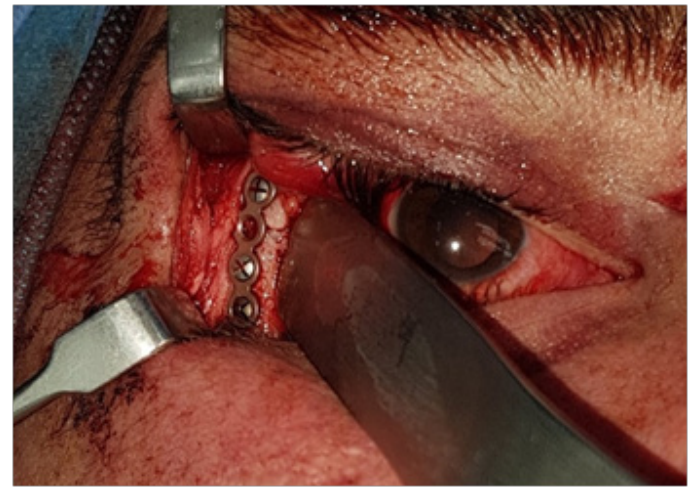

Figure 4 Lateral wall of the right orbit.

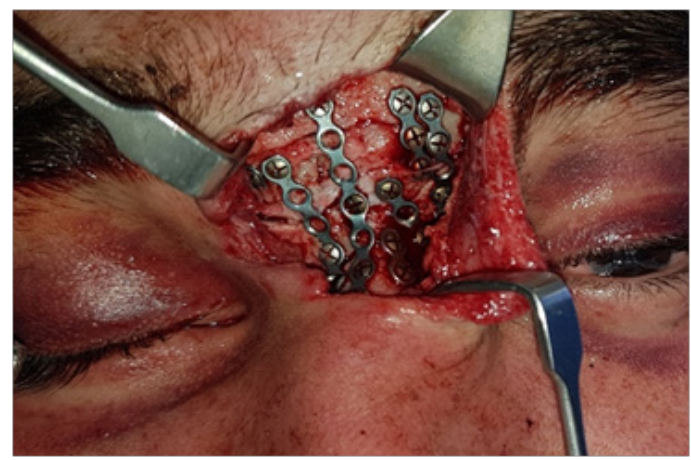

Figure 5 Reductions of the fractured fragments.

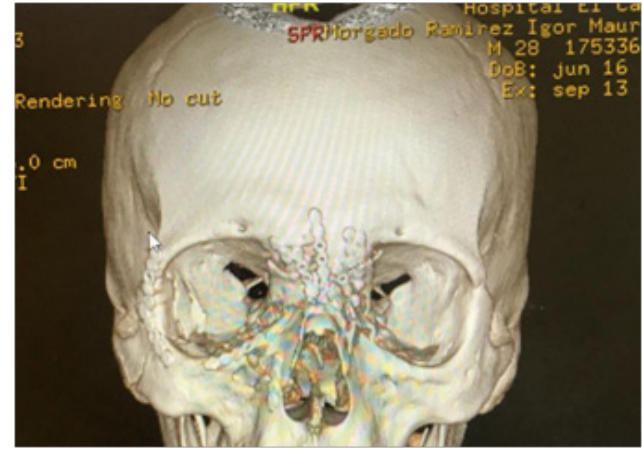

Figure 6 Postoperative analgesic management.

\section{Case 2}

Male patient, 30 years old, in emergency department for functional impotence and increased volume in the upper right limb, added to a nasofrontal contusion due to a fight. The patient is admitted without loss of consciousness, referring to having been assaulted with a blunt object. The patient is evaluated by upper limb trauma team ruling out right arm injury. A CT scan of the facial massif (Figure $7 \& 8$ ) and of the brain was performed, where intra cranial lesion is ruled out and bilateral fracture of the Nasal-Orbit-Ethmoidal and frontal bone is confirmed. After being evaluated by Neurology, Anesthesiology and ophthalmology, the patient is operated to perform the reduction and fixation of his facial fracture through a Coronal approach (Figure $9 \& 10)$. A correct reduction and stabilization of the fragments was achieved by returning the correct anatomy and functionality (Figure 11 \& 12), in addition to an adequate projection of the nasofrontal angle. Finally, the patient remained hospitalized for 48 hours to evaluate evolution and continued periodic check-ups on an outpatient basis. In both cases there were no intra or postoperative complications.

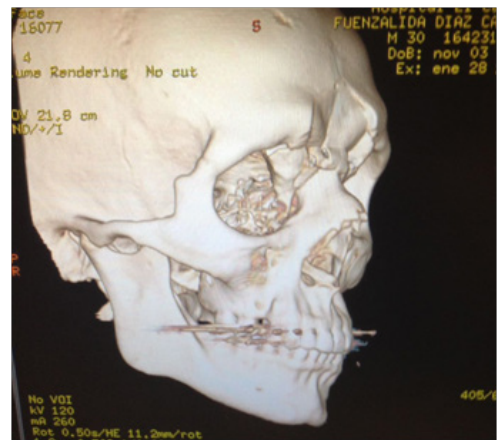

Figure $7 \mathrm{CT}$ scan of the facial massif.

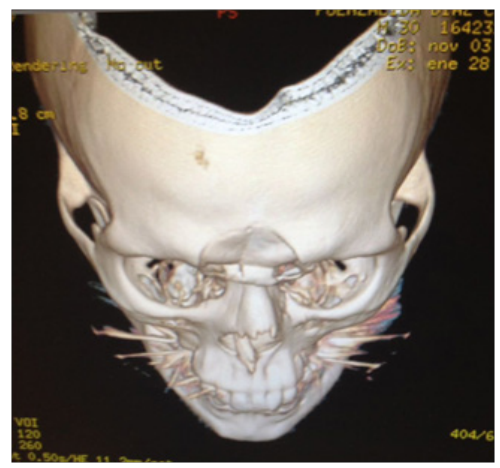

Figure 8 Bilateral fractures of the NOE and frontal bone. 


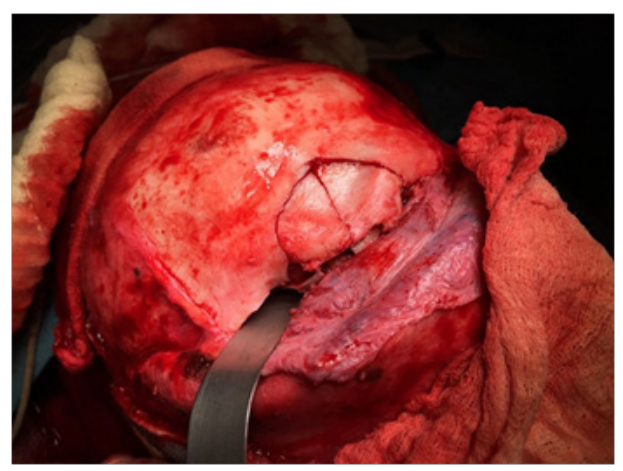

\section{Discussion}

Oral and maxillofacial injuries of traumatic origin are frequent worldwide. In Chile they correspond to $6-8 \%$ of body fractures ${ }^{3}$ and due to their location and aesthetic component they are difficult to handle. ${ }^{4}$ The middle facial third has an important functional and aesthetic component, having an important role in vocal resonance, ocular, olfactory, respiratory and digestive functions, so any alteration in these structures has an aesthetic, functional and psychological impact on the patient. ${ }^{5}$

Nasal-Orbit-Ethmoidal fractures represent $5 \%$ of all facial fractures, the fractures having the lowest incidence in the maxillary facial territory. ${ }^{2}$ The Nasal-Orbit-Ethmoidal complex, corresponds to a complex structure formed by the nasal bones, nasal septum, nasalfrontal process, Ethmoids, Papiraceous plates of the lacrimal bone and sphenoid so any alteration of these structures, will have functional and aesthetic important consequences. The medial canthal ligament corresponds to another structure that is inserted in the frontal process of the maxilla, which in some cases may be compromised to the point of causing disinsertion or displacement of this, causing an increase in the intercanthal distance (traumatic telecanth). ${ }^{5}$

The coronal approach corresponds to the most frequent approach to access this anatomical area and to achieve a good reduction and stabilization of the components of this type of fractures thanks to the high visibility and direct access to the frontal-nasal area and orbital supra arch. ${ }^{4}$ Important to note that while the coronal access provides great visibility and access to this area, it has its limitations in terms of extension since it does not allow us to access the lower part of the medial wall of the orbit, being necessary in many cases to complement this approach with a transconjunctival retro caruncular approach to gain access to this area. ${ }^{6}$ Although the coronal approach is a safe, easy and quick access approach, it is not exempt of complications. Important to consider in this approach leaves an unattractive scar which can be covered by the patient's hair but in those cases of patients with hair less condition, it is important to consider this, making necessary in many cases, the need to seek alternatives to address these area. In the first case, it is observed that the approach to the Fronto-Nasal area through pre-existing lacerations in the area is a viable alternative from the aesthetic point of view since it avoids having to make another incision in the patient, increasing surgical morbidity and the risk of complications of this. In addition to this patient presenting with hair less condition, from the aesthetic point of view it was the best option for the case, thus avoiding a scalp scar. On the other hand, in the second case it is observed that the coronal approach provides excellent access to the fronto-nasal area, facilitating the reduction and fixation of it.

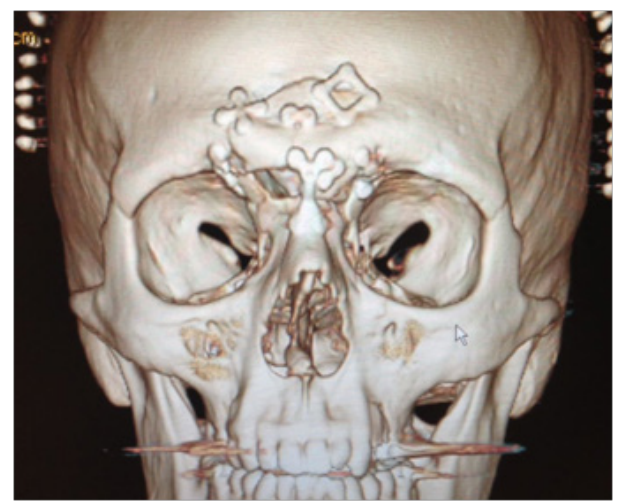

\section{Conclusion}

Maxillofacial trauma based on our experience and as noted above, has a high degree of morbidity, causing functional, aesthetic and psychological alterations to the patient. For this reason, it is that the correct and timely treatment of these lesions allows reducing the complications and sequelae associated with this type of fractures. Although we were able to show that the coronal approach in many cases is the approach of choice for fractures that compromise the Nasal-Orbit-Ethmoidal complex due to its high visibility and access to the area, it has limitations that in some cases, requires the use of pre-lacerations. Existing that is directly related to the fracture area. In these cases, a correct reduction of the fractures was achieved, 
returning adequate functionality and aesthetics to the patient with favorable and aesthetically acceptable results. Although the approach through pre-existing lacerations in the patient is not well documented and there is little evidence of this in the literature, we can conclude that it is a viable option in certain cases where the fracture feature is related to these wounds, thus facilitating access to fractures, when it is well indicated. ${ }^{7-9}$

\section{Funding details}

None.

\section{Acknowledgement}

None.

\section{Conflicts of interest}

The authors declare that there is no conflict of interest.

\section{References}

1. René Rojas S, Gerardo Julián, Jorge Lanking B. Mandibular fractures: experience of a trauma hospital. Rev Medium Chilena. 2002;130(5):30-34.

2. Aracelli Raposoa, Gunther Preislera, Fernando Salinas. Epidemiology of maxillofacial fractures treated surgically in Valdivia, Chile: 5 years of review. Spanish Journal of Oral and Maxillofacial Surgery. 2013;35(1):15-20.
3. Sergio Olate, Sergio Monteiro, Renato Sawazaki. Descriptive Study of Surgical Accesses Used in the Treatment of Fractures of the Orbital Zygomatic Complex. International Journal of Morphology. 2009;27(2):299-304.

4. Edward Ellis, Michael F Zide. Basic Principles for surgical approaches of the Facial Massif. In Surgical Approaches of the Facial Massif (3-6). USA: Lipincott Williams and Wilkins; 2008.

5. Morris CD, Tiwana PS. Diagnosis and Treatment of Midface Fractures. In: Fonseca R, Barber HD, Walker RV, Powers MP, Frost DE, editors. Oral and maxillofacial trauma. St. Louis: Elsevier Inc; 2013.

6. Gerbino G, Zavattero E, Viterbo S, et al. Treatment of Orbital Medial Wall Fractures with Titanium Mesh Plates Using Retrocaruncular Approach: Outcomes with Different Techniques. Craniomaxillofac Trauma Reconstr. 2015;8(4):326-333. Go to:

7. Mast G, Ehrenfeld M, Cornelius CP, et al. Maxillofacial Fractures: Midface and Internal Orbit-Part II: Principles and Surgical Treatment. Facial Plast Surg. 2015;31(4):357-367.

8. Andrés Campolo, Allan Mix, Camila Foncea, et al. Management of maxillofacial trauma in emergency care by non-specialists. Rev Medium Chile. 2017;145(8):1038-1046.

9. Huempfner-Hierl H, Schaller A, Hemprich A, et al. Biomechanical investigation of naso-orbitoethmoid trauma by finite element analysis. $\mathrm{Br} J$ Oral Maxillofac Surg. 2014;52(9):850-853. 